\title{
Resolving GPCR bias
}

\author{
Advances in cryo-electron microscopy combined with improved computational tools for structural modeling have \\ provided new insights into the basis of G-protein-coupled receptor-effector interactions.
}

G -protein-coupled receptors (GPCRs) are the largest known family of membrane receptors, comprising over 800 members that are activated by hormones, lipids, neurotransmitters and peptides. Receptor activation is followed by coupling with downstream effectors such as $G$ proteins and $\beta$-arrestins to promote specific cellular events. Interactions between GPCRs and their effectors can range from broad and promiscuous to highly specific. Efforts over the past two decades focused on determining crystal structures of GPCRs in their ligand-bound states have revealed atomic details regarding binding sites and receptor activation mechanisms. We discussed the existing progress in GPCR structural biology in a 2019 editorial and noted the potential of cryo-electron microscopy (cryo-EM) as an emerging approach for structural studies. At the time, we were hopeful that cryo-EM would reveal molecular details of the interactions of GPCRs with downstream effectors and would answer questions about the mechanistic basis of biased signaling. Since then, major inroads have been made in unraveling the molecular determinants of coupling specificity through structures of ligand-bound GPCRs in complex with multiple $G$ proteins, including in a number of papers published recently in Nature Chemical Biology.

One attractive feature of cryo-EM is that the fast freezing step of sample vitrification preserves the protein conformational state, which is necessary for highly dynamic signaling proteins. This enables the use of engineered $G$ proteins combined with single-chain nanobodies or fusion proteins to stabilize GPCR-G protein complexes. Yet, both crystallography and cryo-EM structures are ultimately only snapshots in time and fail to reveal the full range of meaningful receptor conformations. Molecular dynamics (MD) simulations of structural data can alleviate this shortcoming by offering visualization of global conformational changes associated with receptor activation or inhibition, as exemplified by Cary et al. These authors used a combination of cryo-EM and MD simulations to reveal two distinct agonist-bound GLP-1R conformers occupying distinct regions of an energy

landscape. Computational analyses can serve to generate hypotheses, and can help bridge the gaps between static structures when combined with experimental validation using mutagenesis and other orthogonal approaches.

Cryo-EM has also proven extremely useful for elucidating the molecular determinants of a GPCR's promiscuous interactions with multiple effectors. For example, the class A cholecystokinin receptor subtype CCK1R can couple with a variety of $G$ protein subtypes. Three recent papers (Zhang et al., Liu et al. and Mobbs et al.) have solved cryo-EM structures of ligand-bound CCK1R in complex with $G_{s}, G_{i}$ and $G_{q}$, revealing distinct modes of protein binding and orientation that create variability in the nature of the receptor-G protein interface. For instance, residues in the 'wavy hook' and ICL3 regions of the cholecystokinin receptors are important determinants of $\mathrm{G}$ protein selectivity, providing a structural basis for this promiscuity.

By directing specific GPCR-G protein couplings and downstream effects, biased agonists can reduce the reach of the receptor's otherwise promiscuous interactions. For example, the neuropeptide substance $\mathrm{P}(\mathrm{SP})$ triggers neurokinin1-receptor (NK1R) signaling through $G_{q}$ and $\mathrm{G}_{\mathrm{s}}$ proteins; meanwhile, neurokinin A specifically promotes $G_{q}$ signaling downstream of the same receptor. Harris et al. revealed the cryo-EM structures of NK1R bound to SP or a truncated SP that is $G_{q}$ biased, and found that $G_{s}$ signaling requires interactions between $S P$ and the NK1R extracellular loops. MD simulations revealed that $\mathrm{G}_{\mathrm{q}}$-biased agonists make weaker contacts with these loops.

In addition to directing receptors to a particular $G$ protein, ligands can also direct downstream signaling of the receptor through G-protein- or $\beta$-arrestin-specific paths - the basis of GPCR ligand bias. Shao et al. identified a series of variants of the chemokine CCL15 in which the long forms containing more of the $\mathrm{N}$ terminus bias signaling through the $\beta$-arrestin pathway, whereas shorter forms activate $G$ proteins. Cryo-EM and computational analyses revealed a conformational change of Tyr291 in the CCR1 receptor and alterations in a set of polar residues in the orthosteric binding pocket that underlie this biased agonism.
Similarly, Xu et al. revealed differences in key interactions of the PIF and NPxxY motifs that distinguish the downstream effects of $\mathrm{G}_{\mathrm{i}}$-directing S1PR1 ligand S1P and $\mathrm{G}_{1} / \beta$-arrestin-directing FTY $720-\mathrm{p}$. These papers highlight the idea that GPCRs can be directed toward different downstream outcomes through specific regions and residues of the protein. Future work aimed at defining the general features that dictate ligand bias downstream of GPCRs may lead to therapeutics with improved precision.

Indeed, understanding the structural basis of biased agonism has been believed to better inform drug development by allowing developers to design against inappropriate pathway activation. Medicinal chemistry and large-scale screening approaches are useful for initial identification of hits, but are often of limited selectivity. Recently, the application of in silico or virtual screening using docking simulations has been effective in identifying agonists or antagonists for class A GPCRs, such as an inhibitor for the itch receptor MRGPRX2 and a potent MRGPRX4 agonist. However, the larger orthosteric pockets in class B GPCRs complicate screening. Sutkeviciute et al. developed an approach called essential site scanning analysis to identify residues that undergo conformational changes upon small-molecule binding. Combined with virtual screening, this resulted in the identification of a negative allosteric modulator of the class B GPCR PTH type 1 receptor.

These highlighted papers demonstrate the successful use of cryo-EM approaches to reveal GPCR receptor complexes and will hopefully inspire attempts to revisit understudied GPCRs, such as those in classes B-F, and to define complexes with clinically relevant compounds or unique signaling conformations. In addition, new insights into the structural basis of biased agonism combined with innovations in computational screening will enable the identification of potent and selective modulators of GPCR coupling. With the continuing advances in both GPCR chemical biology and structural determination, we are hopeful that better bridges can be established between these two fields.

Published online: 28 February 2022 https://doi.org/10.1038/s41589-022-00990-Z 